\title{
WHITE BEAM SYNCHROTRON TOPOGRAPHIC CHARACTERISATION OF SILICON WAFERS DIRECTLY BONDED BY OXIDE LAYER
}

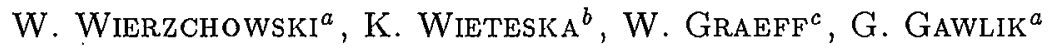 \\ AND M. PAWLOWSKA ${ }^{a}$ \\ ${ }^{a}$ Institute of Electronic Materials Technology, Wólczyńska 133, 01-919 Warsaw, Poland \\ ${ }^{b}$ Institute of Atomic Energy, 05-400 Otwock-Swierk, Poland \\ ${ }^{c}$ HASYLAB at DESY, Notkestraße 85, 22603 Hamburg, Germany
}

Various types of layer structures obtained by direct bonding of oxidised silicon wafers were studied by means of different X-ray topographic methods using white synchrotron beam and the observation of selective etching pattern using scanning electron microscopy and optical microscopy with Nomarski contrast. In the present investigation the particularly important results were obtained with synchrotron section topography, which revealed different defects caused by bonding of thick wafers, in particular the dislocations and microcracks. The different situation was observed in the case of bonding with a very thin layer separated from a silicon substrate by high dose proton implantation. In this case a thin layer accommodated practically all induced strain and the bonded oxidised thick substrate remained defect-free in its inner volume.

PACS numbers: 61.10.Yh, 68.35.-p

\section{Introduction}

The method of direct bonding is an object of intensive studies both in view of its technological applications and new interesting physical phenomena. This methods significantly widens the possibility of manufacturing of new layered structures especially placing of the layers without threading dislocations when the epitaxial growth is extremely difficult.

In the present work the structures obtained by direct bonding of oxidised silicon wafers were studied. These layers are of great perspective application in SOI (silicon on insulator) technology. The application of X-ray methods to bonded oxidised silicon samples was in particular presented by Maszara et al. [1, 2] and Härtwig et al. [3]. 


\section{Experimental}

In the present experiment the three types of multilayer structures obtained by direct bonding of silicon samples with an oxide layer were studied, namely:

1. Structure formed by bonding of two $400 \mu \mathrm{m}$ thick double-side oxidised silicon wafers.

2. Structure made as the previous one, but with major part of one wafer removed by mechanical-chemical polishing and only $40 \mu \mathrm{m}$ of the wafer left.

3. Structure formed by direct bonding of one $400 \mu \mathrm{m}$ double-side oxidised silicon wafer with silicon implanted to a very high dose with $50 \mathrm{keV}$ protons. In this case the implanted crystal is decomposed leaving $0.5 \mu \mathrm{m}$ thick shot-through layer bonded to the oxidised wafer.

All bonded layers were (100) oriented.

The most important results were obtained using synchrotron section topography both in back-reflection and transmission geometry. The synchrotron micro-Laue method and white beam projection topography were simultaneously used in synchrotron experiments.

The Bragg-case section topographs and the micro-Laue method enabled analysis of the mutual disorientation of bonded wafers. If two spots coming from bonded two wafers corresponding to the same reflection are identified the interpretation of their mutual displacement is as follows. The radial component of the displacement is the doubled rotation of the diffraction vector in the plane of diffraction. The tangent component of the displacement is the rotation of the diffraction vector perpendicular to the diffraction plane divided by the cosine of the angle between the direction of actual tangent in the film and the normal to the diffraction plane.

The selective etching pattern was observed with SEM and optical microscope with Nomarski contrast in the second type of samples. The selective etching is otherwise much more difficult at oxidised surfaces, and was not yet successfully performed in other types of presently examined samples.

\section{Results and discussion}

The present investigation revealed a significant difference between the first two types of investigated structures and the third one. In the first two cases the $\mathrm{X}$-ray topographic methods indicated the presence of relatively high concentration of defects in the both wafers forming the investigated structures. This result is in particular illustrated by section topographs shown in Figs. 1 and 2. The defects in these topographs are not well resolved, but these results evidently point their uniformly distributed in the whole thickness of the bonded wafers. In Fig. 1 we may also notice the elements of lattice polygonisation, and some quasi-linear defects.

As may be noticed in Fig. 2 the $40 \mu \mathrm{m}$ thickness of the polished wafer resulted in the much smaller width of the corresponding stripe. The topograph also indicates a relatively high concentration of the defects in the whole thickness of the bonded layer. Differently than the section topograph, the projection topograph shown in Fig. 3 reveals mainly the distribution of defects along the surface. The 


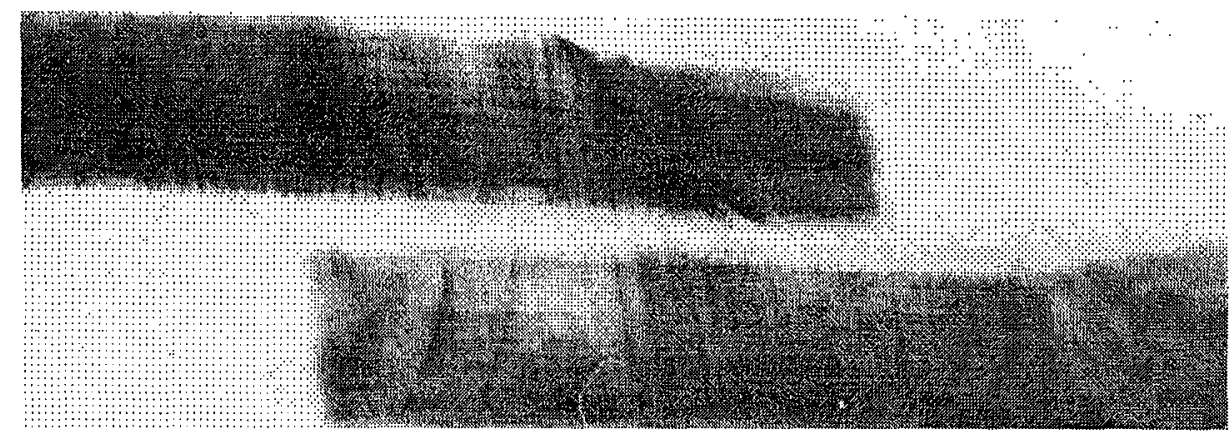

Fig. 1. The representative white beam section topographs of a sample containing two bounded $400 \mu \mathrm{m}$ double sided oxidised wafers. The two stripes correspond to the two bonded layers seen in the same reflection.

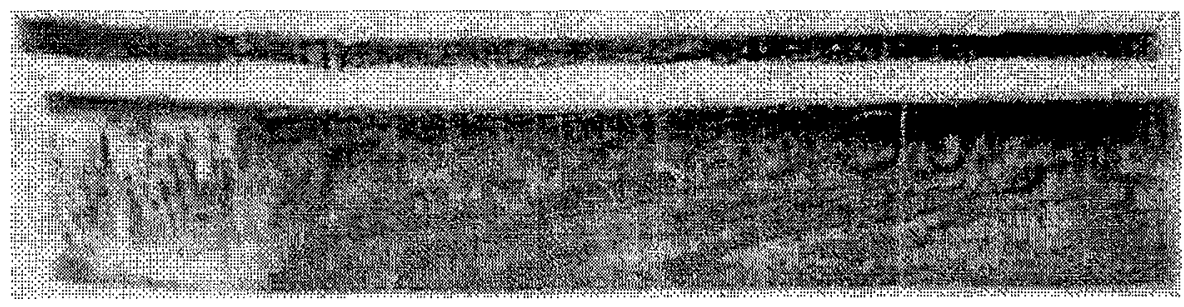

Fig. 2. The Bragg case section topograph of the second type of samples where one of the wafers was reduced to final thickness of $50 \mu \mathrm{m}$. This layer is producing much narrower strip than the substrate.

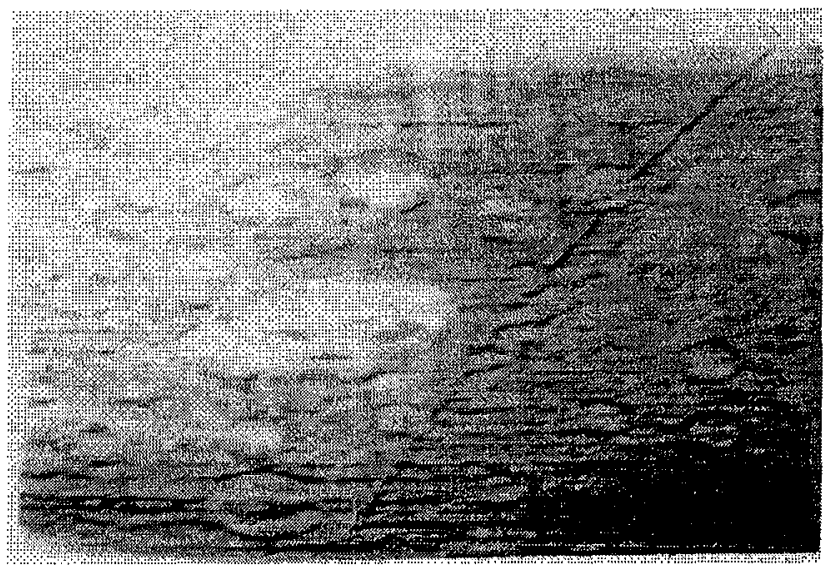

Fig. 3. White beam projection topograph of the sample shown in Fig. 2.

rounded contours may correspond to pressed accidental pollution. The projection topography is on the other hand much less sensitive to various defects than section topography. 

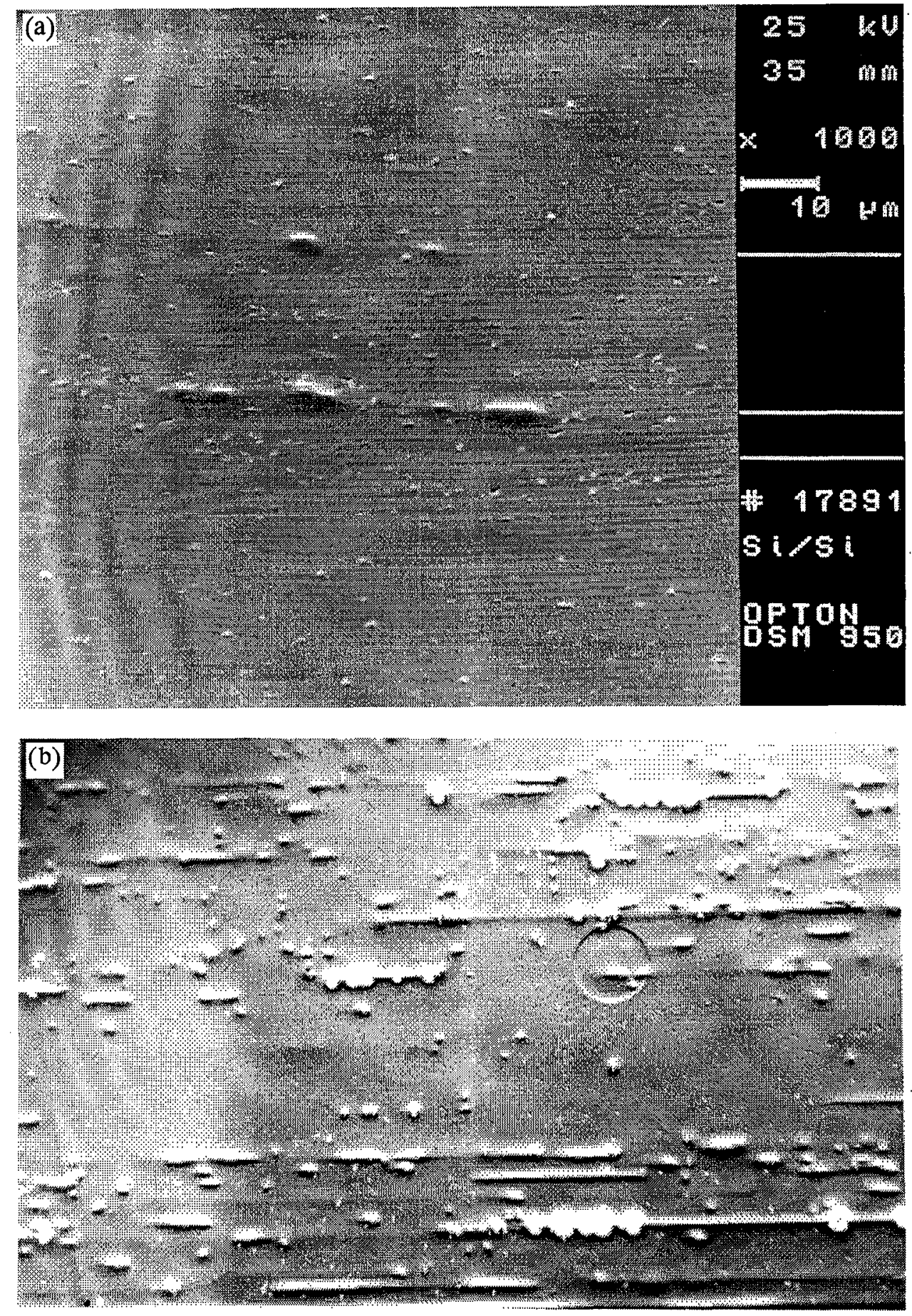

Fig. 4. The selective etching patterns in another fragments of sample shown in Fig. 3 observed using SEM (a) and optical microscope with Nomarski contrast (b). 

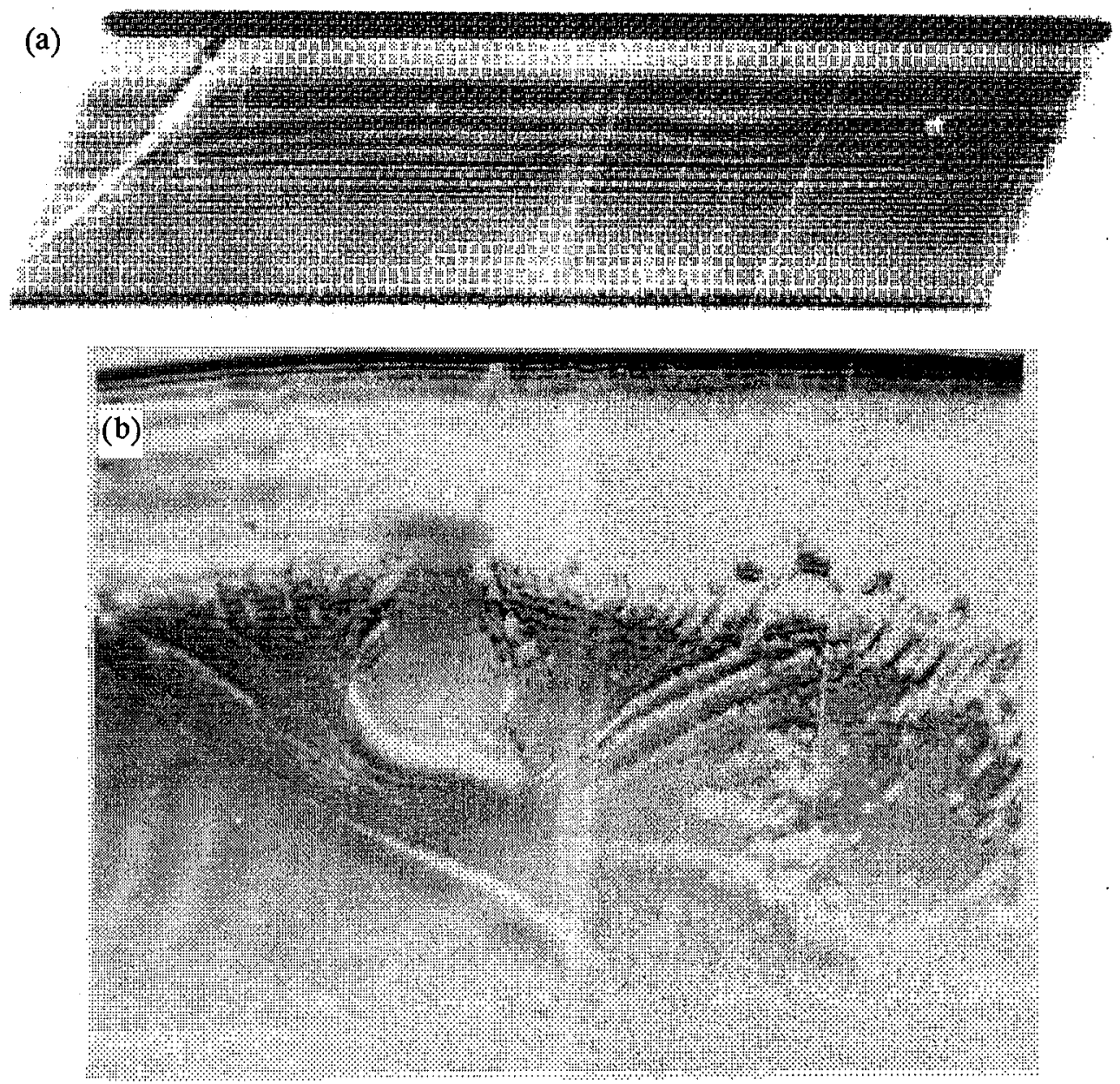

Fig. 5. The Bragg case section topographs of the sample of third type consisting of a $400 \mu \mathrm{m}$ thick double-side oxidised layer bounded with thin proton implantation separated layer. (a) The Bragg-section pattern with a glancing angle close to $10^{\circ}$ exhibiting the interference fringes characteristic of the bent single crystal wafer. (b) The topograph with small glancing angle providing the image of the inhomogeneities of the bonded thin layer.

The high concentration of defects in the bonded samples was confirmed by observation of the selective etching pattern illustrated by Figs. 4a and $b$. The long objects visible in both pictures correspond to the local microcracks. The scanning electron microscope image presents the region with larger concentration of microdefects (i.e. probably small dislocation loops) while in the second picture we may observe a considerable concentration of dislocations. It may be noted that great part of contrast seen in the section topograph corresponds to the microdefects producing shallow etch pits and the visibility of these defects seems to be unusually greater in the present synchrotron experiments. This fact points the 
possible development of dislocation loops connected with microdefects, caused by considerable strain present in the investigated samples.

The other interesting result was obtained using Bragg-case section topographs for the third type of structures with thin layer separated by proton implantation (Fig. 5). In this case the section pattern with a glancing angle close to $10^{\circ}$ exhibited the interference fringes characteristic of the bent single crystal only slightly disturbed by fragments of bonded layer. The topograph with small glancing angle provided the image of the inhomogeneities of the bonded thin layer.

These fringes prove that bonding with thin layer (easy deforming), differently than in previous samples, did not introduce any defect structure.

\section{Conclusions}

The three different types of structures obtained by direct bonding of oxidised silicon layers were studied using the X-ray diffraction methods realised using white synchrotron beam and observation of selective etching patterns.

The very important point of the investigations was the use of Bragg-case synchrotron section topographs. In the case of structures obtained by bonding of thick oxidised layer we observed a large concentration of defects in the whole thickness of the bonded layers. The SEM and Nomarski observations of selective etching pattern contrast indicated presence of microcracks, dislocations and microdefects producing shallow etch pits. The last type of defects is interpreted as due to small dislocation loops.

The bonding with a very thin layer obtained by high dose proton implantation did not introduce the defects to the bonded wafer.

\section{References}

[1] W.P. Maszara, B.-L. Jiang, A. Yamada, G.A. Rozgonyi, H. Baumgart, A.J.R. De Kock, J. Appl. Phys. 69, 257 (1991).

[2] W.P. Maszara, G. Goetz, T. Cavigila, A. Cserhati, G. Johnson, J.B. McKittterick, Mater. Res. Soc. Symp. Proc. 107, 489 (1988).

[3] J. Härtwig, W. Ludwig, M. Ohler, E. Prieur, S. Köhler, C. Guilhalmenc, H. Moriceau, XTOP98 - 4th European Conf. on High Resolution X-Ray Diffraction and Topography, 1998, University of Durham, UK, Programme and Abstracts, P2.19, 1998. 